\title{
Using an FFQ to assess intakes of dietary flavonols and flavones among female adolescents in the Suihua area of northern China
}

\author{
Caihong Sun ${ }^{1}$, Hui Wang ${ }^{2}$, Dong Wang ${ }^{2}$, Yanping Chen ${ }^{3}$, Yan Zhao ${ }^{2}$ and Wei Xia ${ }^{1, *}$ \\ 'Department of Children Health and Hygiene, School of Public Health, Harbin Medical University, 157 Baojian \\ Road, 150081 Harbin, People's Republic of China: ${ }^{2}$ Department of Nutrition and Food Hygiene, School of Public \\ Health, Harbin Medical University, Harbin, People's Republic of China: ${ }^{3}$ Department of Children's Health Care, \\ Harbin Children's Hospital, Harbin, People's Republic of China
}

Submitted 4 June 2013: Final revision received 1 April 2014: Accepted 3 April 2014: First published online 6 May 2014

\begin{abstract}
Objective: The present study aimed to (i) evaluate the reproducibility and validity of a designed FFQ, (ii) apply the FFQ for estimating the dietary intakes of four flavonols and two flavones in female adolescents and (iii) explain their major dietary sources.

Design: The reproducibility between the first and second FFQ administrations (1 year interval) was estimated using the intra-class correlation coefficient. The validity of the first FFQ relative to the average of four three-day $24 \mathrm{~h}$ dietary recalls (24-HR) from four seasons was assessed using the Spearman correlation coefficient. Using a flavonoid content database, the individual flavonol and flavone intakes were calculated and the major food sources were estimated.

Setting: Middle school in Suihua area of Heilongjiang Province, northern China. Subjects: Female adolescents ( $n$ 887) aged $12-18$ years.

Results: Better reproducibility and validity were obtained in the present study. The flavonol and flavone intakes were 16.29 and $4.31 \mathrm{mg} / \mathrm{d}$, respectively. Quercetin and kaempferol were the major contributors $(26.8 \%$ and $23.7 \%$, respectively) to the total intake of flavonols and flavones. The main food sources of flavonols and flavones were apples $(14 \cdot 1 \%)$, followed by potatoes $(7 \cdot 5 \%)$, lettuce $(7 \cdot 3 \%)$ and oranges $(7 \cdot 3 \%)$.

Conclusions: The dietary flavonol and flavone intakes among female adolescents in northern China were similar to those reported in several countries, but significant differences were observed in the food sources ascribed to the geographical location and dietary characteristics.
\end{abstract}

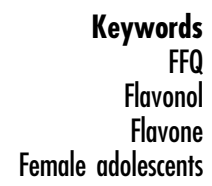

Foods have many components other than the commonly known nutrients such as proteins, fats, carbohydrates, vitamins and minerals, many of which are associated with biological activities related to the reduced risk of several chronic diseases and cancer ${ }^{(1-3)}$. Flavonoids possess a wide range of biochemical and pharmacological effects, including anti-inflammatory and antioxidant effects ${ }^{(4-7)}$. In addition, recent studies have suggested that flavonoid intake may contribute to body weight maintenance in the general female population ${ }^{(8)}$. Flavonol and flavone contents are the highest in plant-based foods, and are concentrated mainly in leafy vegetables and some fruits.

Although flavonoids have various biological activities, their biological function depends mainly on their intakes and bioavailabilities. Databases of flavonoids in Chinese plant foods are currently limited and few data on the estimated flavonoid intake of the Chinese population have been published. Cao et al. ${ }^{(9)}$ reported the major flavonols (quercetin, kaempferol, myricetin and isorhamntin) and flavones (apigenin and luteolin) found in 100 types of vegetables and fruits consumed by the Chinese population. Based on their findings, a preliminary database of flavonols and flavones was built.

Several international studies have reported flavonoid intakes of adults and a few studies have focused on the intake of minor populations, e.g. among the elderly and women ${ }^{(10-12)}$, but few have focused on adolescent girls. Adolescence is an important period of growth and development. Given the special physiological and psychological factors that define adolescence among girls, as well as the phenomenon of blind weight loss, their health has received increasing attention among researchers in recent years ${ }^{(13)}$. Suihua is located in northern China, where the economic condition is not as prosperous as in 
the southern parts of the country. Seasonal vegetables and fruits are still much less eaten, especially during winter. The flavonol and flavone intakes of female adolescents in this area remain unclear. Based on available data on flavonol and flavone contents, the present study was performed to estimate the intakes of two subgroups of flavonoids (flavonols and flavones) and to explain their major dietary sources among female adolescents in the Suihua area in northern China using an FFQ. To demonstrate the feasibility of the FFQ on the target population, the reproducibility and validity of the FFQ were also evaluated. This study would facilitate further investigation on the relationship between flavonoids and chronic diseases.

\section{Methods}

\section{Ethics statement}

The study was approved by the Research Ethics Committee of Harbin Medical University. Written informed consent was obtained from either the participants or their parents (for participants below 18 years old) before the participants were enrolled in the study.

\section{Study population}

Female adolescents aged 12-18 years were enrolled in the study using a stratified multistage cluster sampling method. The participants were randomly recruited from three middle schools in the Suihua area of Heilongjiang Province, China. Each school had six grades. A total of twenty-five resident (living in the countryside) and twentyfive non-resident (living in the city) female students were selected from each grade. A total of 887 adolescents from the 900 potential participants joined in the study, excluding those who did not satisfactorily complete the FFQ ( $n$ 13). Among the qualified participants, 120 girls were expected to participate in the $24 \mathrm{~h}$ dietary recalls (24-HR) and answer the FFQ again after one year.

\section{Study design}

The study began in March 2011 and continued into the following year. Four three-day 24-HR were quarterly collected from 120 participants, who also completed the second FFQ (FFQ2). The first FFQ (FFQ1, $n$ 887) was administered during the first three-day 24-HR, whereas the FFQ2 ( $n$ 120) was administered in February 2012. The reproducibility of the FFQ was estimated using intra-class correlation coefficient (ICC) analysis between FFQ1 and FFQ2, whereas its validity was assessed by comparing FFQ1 with the average of the four three-day 24-HR using Spearman's correlation coefficient.

\section{$F F Q$ and $24 b$ dietary recalls}

The FFQ was developed according to the methodology proposed by Willett ${ }^{(14)}$. Revisions were made to ensure that the list of foods reflected the Chinese diet, according to the 2002 National Health and Dietary Survey ${ }^{(15)}$. Further revisions were made to improve the estimates of flavonoidrich food intakes. The FFQ collected consumption information across eight food groups and eighty-six food items (Table 1). Respondents were requested to recall the average consumption of a given amount of each food item during the previous 1-year period using a graded scale with seven levels, ranging from never to $\geq 3$ times per day. The portion size was also considered for the survey. For the 24-HR, each participant was asked to choose from a set of colour photographs showing different-sized portions of twenty-three specific food items, whereas photographs of dishes, bowls and cups were used to represent the average portion size for the other sixty-three food items. Participants had to describe qualitatively and quantitatively all food consumed during the previous day using the correct

Table 1 Food grouping used in the present study

\begin{tabular}{lll}
\hline Food group & Food items $(n$ 86) & Portion size* \\
\hline Cereals & Steamed bun, bread, corn steamed bun & Number \\
& Baked cake, biscuit & Piece \\
Vegetables & Wheat noodle, fast-food noodle, rice, panicum, purple rice, corn & Bowl \\
& Carrot, white radish, soyabean sprouts, aubergine, cucumber, pumpkin, hot pepper, Chinese cabbage, & Plate \\
& spinach, cabbage, celery, Cucurbita pepo, tomato, potato, sweet potato, onion, parsley, spring onion, \\
& cauliflower, garlic sprouts, garden pea, lettuce, Foeniculum vulgare, potherb mustard, leek, & Number \\
Fruits & mushrooms & Bowl \\
& Apple, orange, pear, banana, grape, watermelon, peach, muskmelon, Chinese pearleaf crabapple, \\
Meat & apricot, hawthorn, plum & Plate \\
Eggs & Date, strawberry, cherry & Number \\
Milk & Pork, beef, mutton, chicken, duck, goose meat, cyprinoid fish, morrhua, shrimp, crab, shellfish & Cup \\
Beans & Chicken egg, duck egg, goose egg, quail egg, pidan, salty egg & Bowl \\
& Milk, yoghurt & Plate \\
& Soyabean, small red bean, green gram, black bean & Tablespoon \\
Drinks & Soyabean curd, dried bean curd, bean products & Cup \\
\hline & Bean paste, Chinese cheese & Cup \\
\hline
\end{tabular}

*Assigned portion sizes for all eighty-six food items used in the FFQ. 
pictures to allow the dietitian to estimate their food intakes. All FFQ data were double entered into a computer and any discrepancies were resolved by referring to the original forms. Two dietitians visited each participant four times to complete the three-day 24-HR and these data were used as a standard to measure the relative validity of the FFQ. The dietitians were instructed to ask the participants key questions for a better qualitative description of food items (e.g. green leafy vegetables and fruits).

\section{Intakes of dietary nutrients, flavonols and flavones}

The nutrient calculator software Fei Hua V2.3 (Institute for Nutrition and Food Security, Chinese Center for Disease Control and Prevention, Beijing, China) was used to calculate the daily intakes of energy and nutrients. The database of flavonols and flavones established by our laboratory was used to calculate the daily flavonol and flavone intakes in our study.

\section{Statistical analysis}

Means and standard deviations of daily energy, flavonol and flavone intakes were calculated for the two FFQ and the average of the four three-day $24-\mathrm{HR}$, and analysed using the Wilcoxon signed-rank test. The residual method was used to help exclude the possibility of variations caused by energy intake $^{(14)}$. Reproducibility between the two FFQ administrations was estimated using ICC ${ }^{(14)}$. The validity of the FFQ relative to the 24-HR was assessed using Spearman's correlation coefficient. Considering within-person variations caused by day-to-day fluctuations and seasonal variations, Spearman's correlation coefficients were 'de-attenuated' using the within- and between-person components of variation from the $24-\mathrm{HR}^{(16)}$. All statistical analyses were performed using the SPSS statistical software package version 13.0 and Microsoft ${ }^{\circledR}$ Excel 2003. Unless stated otherwise, $P<0.05$ was considered significant.

\section{Results}

The mean age and BMI of the participants ( $n$ 887) were $16 \cdot 1$ ( $\mathrm{SD} 1.3$ ) years and 23.9 (SD 3.5$) \mathrm{kg} / \mathrm{m}^{2}$, respectively. The mean intakes of energy, protein, fat, carbohydrate, eight food groups, four flavonols and two flavones of 120 adolescent girls, who completed FFQ1 and four three-day 24-HR from four seasons and FFQ2 for over 1 year, were calculated using the questionnaires as bases, with the aid of the nutrient calculator software and flavonoid database. The aforementioned data are shown in Table 2. The intake of beans from FFQ2 and the intake of drinks from FFQ1 were significantly higher than those from the 24-HR $(P<0.05)$, and the intake of drinks from FFQ2 was lower than that from FFQ1 $(P<0.05)$. No differences were observed for the intakes of energy, protein, fat,

Table 2 Daily mean intakes of energy, macronutrients, food groups, flavonols and flavones of female adolescents aged 12-18 years from the Suihua area of northern China ( $n$ 120), estimated using two FFQ and the average of four three-day $24 \mathrm{~h}$ dietary recalls (24-HR), March 2011-February 2012

\begin{tabular}{|c|c|c|c|c|c|c|}
\hline & \multicolumn{2}{|c|}{ 24-HR } & \multicolumn{2}{|c|}{ FFQ1 } & \multicolumn{2}{|c|}{ FFQ2 } \\
\hline & Mean & SD & Mean & SD & Mean & SD \\
\hline \multicolumn{7}{|l|}{ Macronutrients } \\
\hline Energy (kJ) & 8246 & $1965 \cdot 1$ & 9582 & $2710 \cdot 8$ & 9730 & $3132 \cdot 6$ \\
\hline Protein $(\mathrm{g})$ & 71.56 & $26 \cdot 32$ & 76.02 & 30.72 & $79 \cdot 26$ & 31.54 \\
\hline Fat $(\mathrm{g})$ & 48.67 & 21.06 & 65.69 & 26.45 & 64.23 & 23.52 \\
\hline Carbohydrate $(\mathrm{g})$ & $312 \cdot 14$ & 114.01 & $349 \cdot 26$ & $124 \cdot 28$ & 358.15 & $133 \cdot 13$ \\
\hline \multicolumn{7}{|l|}{ Food groups } \\
\hline Cereals $(\mathrm{g})$ & 433.07 & 110.45 & $462 \cdot 22$ & 121.34 & 476.49 & $148 \cdot 76$ \\
\hline Vegetables (g) & $366 \cdot 34$ & 176.57 & 398.97 & 185.90 & 419.55 & $204 \cdot 45$ \\
\hline Fruits $(\mathrm{g})$ & 259.95 & $126 \cdot 12$ & $276 \cdot 82$ & 153.73 & 295.38 & 140.67 \\
\hline Meat $(\mathrm{g})$ & 57.58 & $16 \cdot 75$ & 63.56 & $27 \cdot 67$ & 59.44 & $27 \cdot 13$ \\
\hline Eggs (g) & 29.94 & $19 \cdot 22$ & 36.54 & 21.43 & 31.59 & 21.94 \\
\hline Milk (g) & 56.43 & 18.63 & $72 \cdot 21$ & 17.35 & 60.85 & 19.29 \\
\hline Beans $(\mathrm{g})$ & $25 \cdot 20$ & 9.47 & 29.42 & $10 \cdot 15$ & $33.42^{*}$ & $16 \cdot 12$ \\
\hline Drinks (ml) & $86 \cdot 21$ & 30.32 & $140.53^{*}$ & 93.10 & $93.35 t$ & 31.53 \\
\hline \multicolumn{7}{|l|}{ Flavonols } \\
\hline Quercetin (mg) & $5 \cdot 17$ & 3.01 & 5.48 & $2 \cdot 81$ & 5.65 & 3.23 \\
\hline Kaempferol (mg) & 4.77 & 1.49 & 5.07 & 2.06 & $5 \cdot 19$ & 2.09 \\
\hline Myricetin (mg) & $2 \cdot 21$ & 1.52 & 2.33 & 1.60 & $2 \cdot 35$ & 1.99 \\
\hline Isorhamntin (mg) & 2.88 & 1.44 & $3 \cdot 10$ & 1.22 & 2.94 & 1.93 \\
\hline Sum of flavonols & 15.03 & 7.48 & $15 \cdot 98$ & $7 \cdot 71$ & $16 \cdot 13$ & $9 \cdot 26$ \\
\hline \multicolumn{7}{|l|}{ Flavones } \\
\hline Luteolin (mg) & $2 \cdot 52$ & 1.78 & $3 \cdot 17$ & 1.80 & 3.02 & 1.22 \\
\hline Apigenin (mg) & 0.96 & 0.68 & 1.05 & 0.51 & 1.09 & 0.65 \\
\hline Sum of flavones & 3.48 & 2.47 & $4 \cdot 22$ & 2.32 & $4 \cdot 11$ & 1.88 \\
\hline Total flavonoids (flavonols + flavones) & 18.51 & 9.98 & $20 \cdot 21$ & $10 \cdot 12$ & $20 \cdot 24$ & $11 \cdot 18$ \\
\hline
\end{tabular}

*Significantly different from 24-HR, Wilcoxon signed-rank test, $P<0.05$. †Significantly different from FFQ1, Wilcoxon signed-rank test, $P<0.05$. 
Table 3 Reproducibility and validity of the FFQ designed for evaluation of the daily flavonol and flavone intakes of female adolescents aged 12-18 years from the Suihua area of northern China, March 2011-February 2012

\begin{tabular}{|c|c|c|c|c|c|}
\hline & \multicolumn{2}{|c|}{ ICC* (FFQ1 v. FFQ2) } & \multicolumn{3}{|c|}{ Spearman's correlation coefficient† (FFQ1 v. 24-HR) } \\
\hline & Crude $\ddagger$ & Energy-adjusted $\ddagger$ & Crude§ & Energy-adjusted§ & De-attenuatec \\
\hline Energy (kJ) & 0.64 & - & 0.65 & - & 0.63 \\
\hline Quercetin (mg) & 0.65 & 0.50 & 0.77 & 0.58 & 0.78 \\
\hline Kaempferol (mg) & 0.50 & 0.44 & 0.56 & 0.41 & 0.59 \\
\hline Myricetin (mg) & 0.47 & 0.38 & 0.63 & 0.43 & 0.65 \\
\hline Isorhamntin (mg) & 0.45 & 0.35 & 0.56 & 0.47 & 0.57 \\
\hline Luteolin (mg) & 0.59 & 0.47 & 0.67 & 0.59 & 0.68 \\
\hline Apigenin (mg) & 0.46 & 0.36 & 0.53 & 0.40 & 0.55 \\
\hline Mean value & 0.52 & 0.42 & 0.62 & 0.48 & 0.64 \\
\hline
\end{tabular}

*Intra-class correlation coefficient (ICC) was used to assess the reproducibility between FFQ1 and FFQ2 administrations ( $n$ 120).

†Spearman's correlation analysis was used to assess the validity of FFQ1 relative to the average of four three-day $24 \mathrm{~h}$ dietary recalls (24-HR; $n$ 120).

$\ddagger$ All ICC values were significant $(P<0.05)$.

$\S$ All Spearman's correlation coefficients were significant $(P<0 \cdot 05)$.

Table 4 Daily flavone and flavonol intakes of female adolescents aged 12-18 years from the Suihua area of northern China, March 2011-February 2012

\begin{tabular}{|c|c|c|c|}
\hline & \multicolumn{2}{|c|}{ Daily intake $(\mathrm{mg})^{*}$} & \multirow[b]{2}{*}{ Percentage of total flavonoids (\%) } \\
\hline & Mean & SD & \\
\hline \multicolumn{4}{|l|}{ Flavonols } \\
\hline Quercetin (mg) & 5.51 & 4.00 & $26 \cdot 8$ \\
\hline Kaempferol (mg) & 5.49 & 3.68 & $23 \cdot 7$ \\
\hline Myricetin (mg) & 2.29 & 1.84 & $11 \cdot 1$ \\
\hline Isorhamntin (mg) & 3.00 & $2 \cdot 37$ & $14 \cdot 6$ \\
\hline Sum of flavonols & $16 \cdot 29$ & 11.91 & $79 \cdot 1$ \\
\hline \multicolumn{4}{|l|}{ Flavones } \\
\hline Luteolin (mg) & $3 \cdot 27$ & 1.63 & $15 \cdot 9$ \\
\hline Apigenin (mg) & 1.03 & 0.58 & $5 \cdot 0$ \\
\hline Sum of flavones & $4 \cdot 31$ & $2 \cdot 21$ & 20.9 \\
\hline Total flavonoids (flavonols + flavones) & $20 \cdot 60$ & $14 \cdot 12$ & 100 \\
\hline
\end{tabular}

*Data from FFQ1 $(n$ 887).

carbohydrate and other food groups derived from the 24-HR, FFQ1 and FFQ2. The mean daily flavonoid intake derived from the 24-HR, FFQ1 and FFQ2 was 18.51 (SD 9.98) $\mathrm{mg} / \mathrm{d}, 20 \cdot 21$ (sD 10.12) $\mathrm{mg} / \mathrm{d}$ and 20.24 (sD 11.18) $\mathrm{mg} / \mathrm{d}$, respectively. No significant differences were detected for the mean total flavonoid intake (flavonols + flavones) and the individual flavonol and flavone intakes.

\section{Reproducibility and relative validity of $F F Q$}

The crude- and energy-adjusted ICC for FFQ1 $v$. FFQ2 were calculated to assess the reproducibility of the FFQ (Table 3). All flavonols and flavones were moderately correlated (ICC $=0 \cdot 4-0 \cdot 7$ ). After adjusting for energy, all ICC decreased. The mean crude ICC was 0.52 from a range of 0.45 (isorhamntin) to 0.65 (quercetin), whereas the mean energy-adjusted ICC was 0.42 from a range of 0.35 (isorhamntin) to 0.50 (quercetin).

The crude, energy-adjusted and de-attenuated Spearman's correlation coefficients for FFQ1 $v$. the average resulting from the four three-day 24-HR are also presented in Table 3 . These values were used to assess the relative validity of the FFQ. The crude Spearman's correlation coefficient between FFQ1 and 24-HR ranged from 0.53 (apigenin) to
0.77 (quercetin), with a mean value of 0.62 . The energyadjusted coefficient ranged from 0.40 (apigenin) to 0.59 (luteolin), with a mean of $0 \cdot 48$. The de-attenuated coefficients ranged from 0.55 (apigenin) to 0.78 (quercetin), with a mean of 0.64. All energy-adjusted Spearman's correlation coefficients were lower than the crude ICC in terms of FFQ1 and FFQ2. However, de-attenuation to correct for intraindividual variability improved the Spearman's correlation coefficients and led to an increase in the mean Spearman's correlation coefficient for FFQ1 v. 24-HR (from 0.62 to 0.64).

\section{Estimated flavonol and flavone intakes and major food sources}

The mean daily dietary flavonol and flavone intakes were estimated from the FFQ (FFQ1, $n$ 887) completed by female adolescents in the Suihua area of northern China. The total flavonoid intake (flavonols + flavones) was $20 \cdot 60$ (SD 14.12) $\mathrm{mg} / \mathrm{d}$, mainly from quercetin and kaempferol (26.8\% and $23.7 \%$, respectively), followed by luteolin (15.9\%), isorhamntin (14.6\%) and myricetin (11.1\%). The contribution of apigenin was relatively minimal $(5 \cdot 0 \%)$. The flavonol intake was 16.29 (sD 11.91) $\mathrm{mg} / \mathrm{d}(79 \cdot 1 \%)$ and the flavone intake was $4 \cdot 31$ (SD 2.21) $\mathrm{mg} / \mathrm{d}(20 \cdot 9 \%$; Table 4). 
Table 5 Main food sources and their contribution (\%) to the flavone and flavonol intakes of female adolescents aged 12-18 years from the Suihua area of northern China, March 2011-February 2012

\begin{tabular}{|c|c|c|c|c|c|c|c|}
\hline \multirow[b]{2}{*}{ Food } & \multicolumn{4}{|c|}{ Flavonols* } & \multicolumn{2}{|c|}{ Flavonols* } & \multirow[b]{2}{*}{$\% \dagger$} \\
\hline & Quercetin & Kaempferol & Myricetin & Isorhamntin & Luteolin & Apigenin & \\
\hline Apple & $30 \cdot 4$ & $15 \cdot 0$ & & & & & $11 \cdot 7$ \\
\hline Lettuce & $27 \cdot 3$ & & & & & & $7 \cdot 3$ \\
\hline Orange & $16 \cdot 8$ & & & $17 \cdot 1$ & & & $7 \cdot 0$ \\
\hline Muskmelon & $10 \cdot 5$ & & & & & & $2 \cdot 8$ \\
\hline Potato & & $41 \cdot 6$ & & & & & 9.9 \\
\hline Soyabean sprouts & & $17 \cdot 7$ & & & & & $4 \cdot 2$ \\
\hline Leek & & $16 \cdot 3$ & & & & & 3.9 \\
\hline Hawthorn & & & $26 \cdot 0$ & & & & $3 \cdot 2$ \\
\hline Celery & & & $24 \cdot 3$ & & & 30.5 & 4.2 \\
\hline Strawberry & & & 14.7 & & & & 1.8 \\
\hline Cherry & & & $12 \cdot 6$ & & & & 1.6 \\
\hline Chinese cabbage & & & & $32 \cdot 3$ & & & 4.7 \\
\hline Potherb mustard & & & & $10 \cdot 4$ & & & 1.6 \\
\hline Pumpkin & & & & $10 \cdot 0$ & & & 1.5 \\
\hline Tomato & & & & & $26 \cdot 2$ & & 4.2 \\
\hline Aubergine & & & & & $24 \cdot 4$ & & 3.9 \\
\hline White radish & & & & & $19 \cdot 2$ & & $3 \cdot 3$ \\
\hline Garlic sprouts & & & & & $13 \cdot 8$ & & $2 \cdot 3$ \\
\hline Sweet potato & & & & & & $16 \cdot 2$ & 1.3 \\
\hline Peach & & & & & & $10 \cdot 9$ & 0.9 \\
\hline Onion & & & & & & $8 \cdot 8$ & 0.7 \\
\hline Others & $15 \cdot 0$ & 9.4 & $22 \cdot 4$ & $30 \cdot 2$ & 28.4 & 33.6 & $17 \cdot 8$ \\
\hline
\end{tabular}

*Percentage of individual flavonols or flavones.

†Percentage of total flavonols and flavones.

The primary food items contributing to the individual flavonol and flavone intakes are shown in Table 5. After colligating the daily dietary intakes, the results show that apple was the primary food item contributing to the total flavonol and flavone intakes (11.7\%), followed by potatoes $(9.9 \%)$ and lettuce $(7.3 \%)$. Other main contributors included oranges $(7.0 \%)$, Chinese cabbage ( $4.7 \%$ ), tomatoes $(4.2 \%)$, celery ( $4.2 \%)$, soyabean sprouts ( $4.2 \%)$, leeks (3.9\%) and aubergine (3.9\%). The sources of the total flavonoid intakes (flavonols + flavones) were vegetables $(55.6 \%)$, fruits $(26.6 \%)$ and others $(17.8 \%$; e.g. cereals, tea, fruit juice and coffee).

\section{Discussion}

Given that the FFQ method is inexpensive and reflects the long-term dietary conditions of participants, it is generally considered to be a good tool for conducting dietary nutritional epidemiological studies. The results from FFQ differ depending on cultural background, eating habits, food sources, season, climate, age, economy and other factors, even in the same country. Thus, a special FFQ should be designed for the target population while considering the differences in food items that contribute to the daily supply of nutrients or phytochemicals with biological activities $^{(17)}$. Suihua is located in north-east China, where winter lasts for more than five months and the economic condition is not as prosperous as that of the southern part of the country. In the current study, a new FFQ was designed for adolescent girls from Suihua, with the specific aim of selecting food groups that contribute significantly to flavonol and flavone intakes.

The reproducibility and relative validity of the FFQ were evaluated. The crude and energy-adjusted ICC for the mean daily flavonol and flavone intakes from FFQ1 $v$. FFQ2 were 0.52 and 0.42 , respectively, for reproducibility. The crude, energy-adjusted and de-attenuated Spearman's correlation coefficients for the mean daily flavonol and flavone intakes from FFQ1 v. 24-HR were 0.62, 0.48 and 0.64 , respectively, for validity, indicating that better correlations were obtained. Our study population consisted of a group of female adolescents with similar lifestyles (i.e. students in three middle schools who frequently dine in the school canteen), which possibly contributed to the moderate correlations. The ability of an individual to deal with abstract concepts and form mental images of her dietary regimen that are as close as possible to the truth $^{(18)}$, was another crucial factor in producing reliable estimates of habitual intake using the FFQ. Thus, colour photographs of foods taken in classrooms were used to make the task less tedious and encourage the interviewees to provide more accurate dietary information ${ }^{(19)}$. When the 24-HR were used to estimate the individual dietary intakes, we used a method that provided better estimates of the intake of even episodically consumed foods by accounting for the correlation between the probability of consumption and the amount consumed, and incorporating covariate information ${ }^{(20,21)}$. Adjusting for energy decreased the correlation in almost all subclasses of 
flavonoids, as high-energy foods may contain little to no amounts of flavonols and flavones ${ }^{(22)}$.

Despite numerous reports on the health-improving effects of flavonoids, a limited number of studies on flavonoid intake are available worldwide. The flavonol and flavone intakes from several countries are listed in Table 6, showing that the estimates varied widely among studies. In the current study, the mean flavonoid (four flavonols and two flavones) intake of 887 female adolescents was $20 \cdot 6 \mathrm{mg} / \mathrm{d}$. The total flavonol intake was $16.3 \mathrm{mg} / \mathrm{d}$ and the total flavone intake was $4.3 \mathrm{mg} / \mathrm{d}$. These results are similar to those in studies conducted in Australia ${ }^{(23)}$, the Netherlands ${ }^{(24)}$, China $^{(25)}$ and the USA ${ }^{(26)}$, and are even higher than those in other studies reported from the USA ${ }^{(27,28)}$, Finland ${ }^{(11,29)}$ and $J_{a p a n}{ }^{(30,31)}$. However, our results are lower than those found in studies conducted in Belgium ${ }^{(22)}$ and Spain ${ }^{(32)}$.

The major dietary sources of flavonol and flavone intakes reported in some of the aforementioned countries are onions and tea, followed by apples, broccoli and lettuce $^{(24,26,33)}$. In the present study, apples, potatoes, lettuce, oranges, soyabean sprouts and leeks were the main food sources of flavonols, whereas tomatoes, aubergine, white radishes, celery and sweet potatoes were the main sources of flavones, similar to the results of a study on the Australian population with a sample population aged $16-18$ years ${ }^{(23)}$. The participants in our study were adolescent girls with special dietary characteristics and much lower intakes of tea and onions compared with the respective populations of Western countries, leading to differences in both intake amounts and major sources. The contributions of cereals and cereal-containing foods, which the girls consumed in large amounts, to the flavonol and flavone intakes were minimal (7.2\%) in the present study. The differences in results could also be explained by the potato consumption. Our participants living in the Suihua area partly ate potatoes instead of the staple food, e.g. rice, making potato the primary food item that subsequently contributed to the total flavonol and flavone intake.

The current study had several limitations. First, only two subgroups of flavonoids were studied. However, other subclasses of flavonoids, such as isoflavones and isoflavanones, are also consumed in high amounts in China. This limitation could be ascribed to the flavonoid database, which has a limited number of food items, although the database is constantly being updated. Therefore, broader evaluations on other subclasses of flavonoids must be conducted. The second limitation was the time for the FFQ to be completed. The flavonoid contents of foods are influenced by region, season, sunlight and other factors ${ }^{(24)}$. For instance, the quercetin content of onions measured in our laboratory was $1.23 \mathrm{mg} / 100 \mathrm{~g}$ (fresh weight), which was much lower than that in the US flavonoid database $(7.29-33.43 \mathrm{mg} / 100 \mathrm{~g}$, fresh weight) and the contents reported by Arabbi et al. $\left(38.3-93.6 \mathrm{mg} / 100 \mathrm{~g}\right.$, fresh weight ${ }^{(34)}$. Third, the content changes in the storage and processing of the foods were not considered. As previously mentioned, food storage

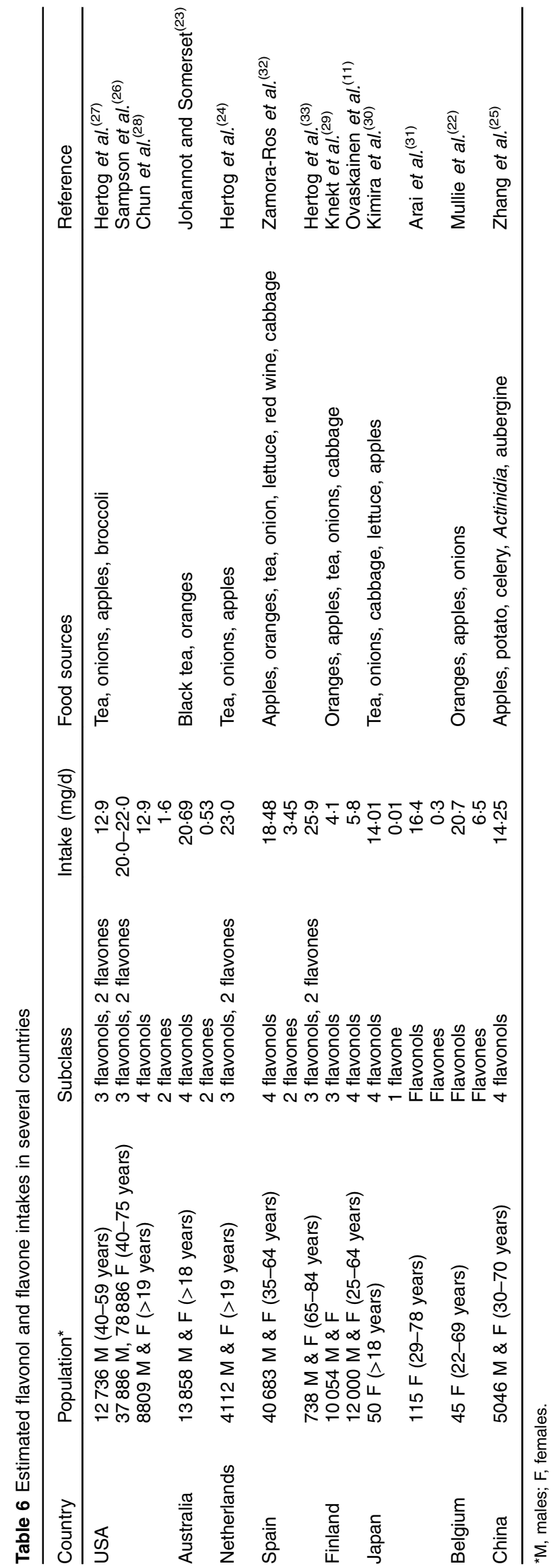


temperature, processing and peeling can influence flavonoid content $^{(35)}$. Thus, more in-depth studies must be performed.

\section{Conclusion}

In conclusion, based on the proven reproducibility and validity of the FFQ, the present study estimated the dietary intakes and sources of flavonols and flavones among female adolescents. The dietary flavonoid intakes among female adolescents in the Suihua area were similar to those reported in previous studies, even though winter is longer and the economy is relatively poor in this area. The food sources showed significant differences because of the geographical position and dietary characteristics. This work provides data for epidemiological studies on the proposed relationship between these flavonoids and human health.

\section{Acknowledgements}

Financial support: This study was supported by a grant from the Science and Technology Agency of Heilongjiang Province, China (Youth Fund to W.X., QC06C059). The funder, W.X., contributed to the study design, conduct of the study, analysis of samples or data, interpretation of findings or the preparation of the manuscript. Conflict of interest: None. Authorship: W.X. conceived and designed the study, and is responsible for final editing and approval of the manuscript. C.S., H.W., D.Z. and Y.C. performed the investigations. C.S. and Y.Z. analysed the data and wrote the manuscript. All authors approved the final version of the paper for publication. Ethics of human subject participation: This study was approved by the Research Ethics Committee of Harbin Medical University. Written informed consent was obtained from either the participants or their parents before they were enrolled in the study.

\section{References}

1. Craig WJ (2010) Nutrition concerns and health effects of vegetarian diets. Nutr Clin Pract 25, 613-620.

2. Alvarez-Parrilla E, De La Rosa LA, Legarreta P et al. (2010) Daily consumption of apple, pear and orange juice differently affects plasma lipids and antioxidant capacity of smoking and non-smoking adults. Int J Food Sci Nutr 61, 369-380.

3. Hung H (2007) Dietary quercetin inhibits proliferation of lung carcinoma cells. Forum Nutr 60, 146-157.

4. Beecher GR (2003) Overview of dietary flavonoids: nomenclature, occurrence and intake. J Nutr 133, issue 10, 3248S-3254S.

5. Blumberg J (2003) Introduction to the proceedings of the Third International Scientific Symposium on Tea and Human Health: Role of Flavonoids in the Diet. J Nutr 133, issue 10,3244 S-3246S.

6. Hollman PC \& Katan MB (1999) Dietary flavonoids: intake, health effects and bioavailability. Food Chem Toxicol 37, 937-942.

7. Prior RL \& Cao G (1999) Antioxidant capacity and polyphenolic components of teas: implications for altering in vivo antioxidant status. Proc Soc Exp Biol Med 220, 255-261.
8. Hughes LA, Arts IC, Ambergen T et al. (2008) Higher dietary flavone, flavonol, and catechin intakes are associated with less of an increase in BMI over time in women: a longitudinal analysis from the Netherlands Cohort Study. Am J Clin Nutr 88, 1341-1352.

9. Cao J, Chen W, Zhang Y et al. (2010) Content of selected flavonoids in 100 edible vegetables and fruits. Food Sci Technol Res 16, 395-402.

10. Chun OK, Chung SJ \& Song WO (2007) Estimated dietary flavonoid intake and major food sources of US adults. J Nutr 137, 1244-1252.

11. Ovaskainen ML, Torronen R, Koponen JM et al. (2008) Dietary intake and major food sources of polyphenols in Finnish adults. J Nutr 138, 562-566.

12. Otaki N, Kimira M, Katsumata S et al. (2009) Distribution and major sources of flavonoid intakes in the middle-aged Japanese women. J Clin Biochem Nutr 44, 231-238.

13. Johnson F, Wardle J \& Griffith J (2002) The adolescent food habits checklist: reliability and validity of a measure of healthy eating behaviour in adolescents. Eur J Clin Nutr 56, 644-649.

14. Willett W (1987) Nutritional epidemiology: issues and challenges. Int J Epidemiol 16, 312-317.

15. Li LM, Rao KQ, Kong LZ et al. (2005) A description on the Chinese national nutrition and health survey in 2002. Zhonghua Liu Xing Bing Xue Za Zhi 26, 478-484.

16. Rosner B \& Willett WC (1988) Interval estimates for correlation coefficients corrected for within-person variation: implications for study design and hypothesis testing. Am J Epidemiol 127, 377-386.

17. Zhang CX \& Ho SC (2009) Validity and reproducibility of a food frequency questionnaire among Chinese women in Guangdong province. Asia Pac J Clin Nutr 18, 240-250.

18. Yaroch AL \& Resnicow K (2000) Development of a modified picture-sort food frequency questionnaire administered to low-income, overweight, African-American adolescent girls. J Am Diet Assoc 100, 1050-1056.

19. Tooze JA, Midthune D, Dodd KW et al. (2006) A new statistical method for estimating the usual intake of episodically consumed foods with application to their distribution. $J \mathrm{Am}$ Diet Assoc 106, 1575-1587.

20. Souverein OW, Dekkers AL, Geelen A et al. (2011) Comparing four methods to estimate usual intake distributions. Eur J Clin Nutr 65, Suppl. 1, S92-S101.

21. Goodwin RA, Brule D, Junkins EA et al. (2001) Development of a food and activity record and a portion-size model booklet for use by 6- to 17-year olds: a review of focus-group testing. J Am Diet Assoc 101, 926-928.

22. Mullie P, Clarys P, Deriemaeker P et al. (2008) Estimation of daily human intake of food flavonoids. Int J Food Sci Nutr 59, 291-298.

23. Johannot L \& Somerset SM (2006) Age-related variations in flavonoid intake and sources in the Australian population. Public Health Nutr 9, 1045-1054.

24. Hertog MG, Hollman PC, Katan MB et al. (1993) Intake of potentially anticarcinogenic flavonoids and their determinants in adults in The Netherlands. Nutr Cancer 20, 21-29.

25. Zhang Y, Li Y, Cao C et al. (2010) Dietary flavonol and flavone intakes and their major food sources in Chinese adults. Nutr Cancer 62, 1120-1127.

26. Sampson L, Rimm E, Hollman PC et al. (2002) Flavonol and flavone intakes in US health professionals. J Am Diet Assoc 102, 1414-1420.

27. Hertog MG, Kromhout D, Aravanis C et al. (1995) Flavonoid intake and long-term risk of coronary heart disease and cancer in the seven countries study. Arch Intern Med $\mathbf{1 5 5}$, 381-386.

28. Chun OK, Floegel A, Chung SJ et al. (2010) Estimation of antioxidant intakes from diet and supplements in US adults. J Nutr 140, 317-324. 
29. Knekt P, Kumpulainen J, Jarvinen R et al. (2002) Flavonoid intake and risk of chronic diseases. Am J Clin Nutr $\mathbf{7 6}$, 560-568.

30. Kimira M, Arai Y, Shimoi K et al. (1998) Japanese intake of flavonoids and isoflavonoids from foods. J Epidemiol 8, 168-175.

31. Arai Y, Watanabe S, Kimira M et al. (2000) Dietary intakes of flavonols, flavones and isoflavones by Japanese women and the inverse correlation between quercetin intake and plasma LDL cholesterol concentration. J Nutr 130, 2243-2250.

32. Zamora-Ros R, Andres-Lacueva C, Lamuela-Raventos RM et al. (2010) Estimation of dietary sources and flavonoid intake in a Spanish adult population (EPIC-Spain). J Am Diet Assoc 110, 390-398.

33. Hertog MG, Feskens EJ, Hollman PC et al. (1994) Dietary flavonoids and cancer risk in the Zutphen Elderly Study. Nutr Cancer 22, 175-184.

34. Arabbi PR, Genovese MI \& Lajolo FM (2004) Flavonoids in vegetable foods commonly consumed in Brazil and estimated ingestion by the Brazilian population. J Agric Food Chem 52, 1124-1131.

35. Srivastava A, Akoh CC, Yi W et al. (2007) Effect of storage conditions on the biological activity of phenolic compounds of blueberry extract packed in glass bottles. J Agric Food Chem 55, 2705-2713. 